\title{
Front Matter: Volume 10763
}

, "Front Matter: Volume 10763," Proc. SPIE 10763, Radiation Detectors in Medicine, Industry, and National Security XIX, 1076301 (8 November 2018); doi: $10.1117 / 12.2516196$

EDIE Event: SPIE Optical Engineering + Applications, 2018, San Diego, California, SPIE. United States 


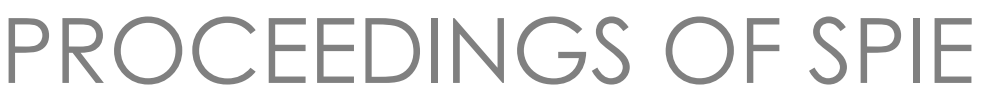

\title{
Radiation Detectors in Medicine, Industry, and National Security XIX
}

\author{
Gary P. Grim
}

Lars R. Furenlid

H. Bradford Barber

Editors

22-23 August 2018

San Diego, California, United States

Sponsored and Published by

SPIE 
The papers in this volume were part of the technical conference cited on the cover and title page. Papers were selected and subject to review by the editors and conference program committee. Some conference presentations may not be available for publication. Additional papers and presentation recordings may be available online in the SPIE Digital Library at SPIEDigitalLibrary.org.

The papers reflect the work and thoughts of the authors and are published herein as submitted. The publisher is not responsible for the validity of the information or for any outcomes resulting from reliance thereon.

Please use the following format to cite material from these proceedings:

Author(s), "Title of Paper," in Radiation Detectors in Medicine, Industry, and National Security XIX, edited by Gary P. Grim, Lars R. Furenlid, H. Bradford Barber, Proceedings of SPIE Vol. 10763 (SPIE, Bellingham, WA, 2018) Seven-digit Article CID Number.

ISSN: 0277-786X

ISSN: 1996-756X (electronic)

ISBN: 9781510620971

ISBN: 9781510620988 (electronic)

Published by

SPIE

P.O. Box 10, Bellingham, Washington 98227-0010 USA

Telephone +1 3606763290 (Pacific Time) · Fax +1 3606471445

SPIE.org

Copyright (C) 2018, Society of Photo-Optical Instrumentation Engineers.

Copying of material in this book for internal or personal use, or for the internal or personal use of specific clients, beyond the fair use provisions granted by the U.S. Copyright Law is authorized by SPIE subject to payment of copying fees. The Transactional Reporting Service base fee for this volume is $\$ 18.00$ per article (or portion thereof), which should be paid directly to the Copyright Clearance Center (CCC), 222 Rosewood Drive, Danvers, MA 01923. Payment may also be made electronically through CCC Online at copyright.com. Other copying for republication, resale, advertising or promotion, or any form of systematic or multiple reproduction of any material in this book is prohibited except with permission in writing from the publisher. The CCC fee code is 0277$786 \mathrm{X} / 18 / \$ 18.00$.

Printed in the United States of America.

Publication of record for individual papers is online in the SPIE Digital Library.

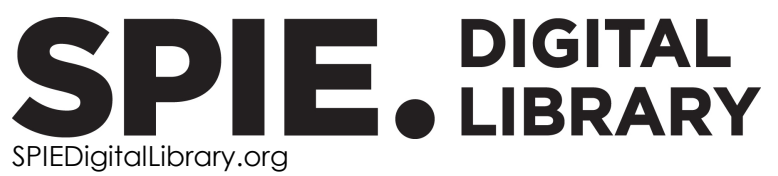

Paper Numbering: Proceedings of SPIE follow an e-First publication model. A unique citation identifier (CID) number is assigned to each article at the time of publication. Utilization of CIDs allows articles to be fully citable as soon as they are published online, and connects the same identifier to all online and print versions of the publication. SPIE uses a seven-digit CID article numbering system structured as follows:

- The first five digits correspond to the SPIE volume number.

- The last two digits indicate publication order within the volume using a Base 36 numbering system employing both numerals and letters. These two-number sets start with $00,01,02,03,04$, 05, 06, 07, 08, 09, 0A, OB ... 0Z, followed by 10-1Z, 20-2Z, etc. The CID Number appears on each page of the manuscript. 


\title{
Contents
}

\author{
vii Authors \\ ix Conference Committee
}

SESSION 1 MEDICAL APPLICATIONS: PET/SPECT

1076302 Dual-energy $\mathrm{x}$-ray computed tomography scanner using a room-temperature cadmiumtelluride detector and a range-region counter [10763-1]

1076303 lonizing radiation induces femtosecond time scale modulations of a material's optical properties (Invited Paper) [10763-2]

1076304 System integration for a SPECT system featuring digital waveform acquisition [10763-3]

1076305 Parameters optimization of volumetric reconstruction technique for L-SPECT imaging [10763-4]

1076306 Scintillator-specific optimization of 2-bit sigma-delta modulation A/D for gamma-ray pulse waveform capture [10763-5]

\section{SESSION 2 SECURITY AND DEFENSE APPLICATIONS}

1076308 Application of International Radiological Information Exchange (IRIX) standards for radiation monitoring data reporting (Invited Paper) [10763-7]

1076309 Harmonization of radiation monitoring and environmental sampling data in the aftermath of a nuclear or radiological emergency [10763-9]

$107630 \mathrm{~A} \quad$ New energy spectral measurements of a distributed $x$-ray source with a Compton spectrometer [10763-10]

\section{SESSION 3 MEDICAL APPLICATIONS: RADIOGRAPHY/CT I}

10763 OC Demonstration of a high resolution x-ray detector for medical imaging [10763-11]

10763 OD Bridgman-grown Csl:Tl crystals codoped to suppress afterglow for high-throughput cargo inspection (Invited Paper) [10763-12]

10763 OE Digital image capture for high-resolution medical x-ray diagnostics [10763-13] 
10763 OF Non-destructive examination of additive manufactured acetabular hip prosthesis cups (Invited Paper) [10763-14]

\section{SESSION 4 MEDICAL APPLICATIONS: RADIOGRAPHY/CT II}

$107630 \mathrm{G} \quad$ Unsupervised learning methods to perform material identification tasks on spectral computed tomography data (Invited Paper) [10763-15]

$10763 \mathrm{OH} \quad$ Triple-energy high-count-rate x-ray computed tomography scanner using a cooled cadmiumtelluride detector [10763-16]

$10763 \mathrm{Ol} \quad$ Dual-energy high-count-rate x-ray computed tomography using a cerium-doped yttriumaluminum-perovskite crystal and a photomultiplier tube [10763-18]

\section{SESSION $5 \quad$ ICF DIAGNOSTICS: FAST X-RAY IMAGING}

$10763 \mathrm{OL} \quad$ Performance characterization of a four-frame nanosecond gated hybrid CMOS image sensor [10763-20]

10763 OM Design and characterization of a novel 1-ns multi-frame imager for the Ultra-Fast X-ray Imager (UXI) program at Sandia National Laboratories [10763-21]

10763 ON Design of radiation tolerant FPGAs for the LLNL nanosecond gated CMOS camera program [10763-22]

\section{SESSION $6 \quad$ ICF DIAGNOSTICS: NEUTRON, GAMMA, AND OTHER}

10763 OP Lens design challenges for scintillator-based neutron imaging (Invited Paper) [10763-24]

$107630 Q \quad$ Evolution of the neutron imaging aperture [10763-25]

10763 OS Debris studies for high-repetition rate and high-power laser experiments at the Central Laser Facility [10763-27]

\section{SESSION 7 ICF DIAGNOSTICS: INSTRUMENTATION}

10763 OU Coded aperture x-ray imaging of high power laser-plasma interactions on the Vulcan Laser System [10763-29]

10763 OV Capacitor transient effects in a high-dose-rate environment [10763-32] 
POSTER SESSION

10763 OX SEPAGE: a proton-ion-electron spectrometer for LMJ-PETAL [10763-33]

Proc. of SPIE Vol. $107631076301-5$

Downloaded From: https://www.spiedigitallibrary.org/conference-proceedings-of-spie on 26 Apr 2023 Terms of Use: https://www.spiedigitallibrary.org/terms-of-use 
Proc. of SPIE Vol. 10763 1076301-6

Downloaded From: https://www.spiedigitallibrary.org/conference-proceedings-of-spie on 26 Apr 2023 Terms of Use: https://www.spiedigitallibrary.org/terms-of-use 


\section{Authors}

Numbers in the index correspond to the last two digits of the seven-digit citation identifier (CID) article numbering system used in Proceedings of SPIE. The first five digits reflect the volume number. Base 36 numbering is employed for the last two digits and indicates the order of articles within the volume. Numbers start with 00, 01, 02, 03, 04, 05, 06, 07, 08, 09, OA, OB...0Z, followed by 10-1Z, 20-2Z, etc.

Adams, Bernhard, OC, OE

Akif, Adil, OC, OE

Albinet, Franck, 08

Anderson-Asubonteng, A., OU

Astbury, S., OS

Attridge, Alex, OF

Baciu, Florian, 08, 09

Batani, Dimitri, OX

Beach, M., OV

Bell, P., OV

Bhattacharya, P., OD

Bifano, Thomas, OC, OE

Boone, A., OM

Booth, N., OS, OU

Boutoux, Guillaume, OX

Brecher, C., OD

Bryce, E., OS

Caillaud, Tony, OX

Carpenter, Arthur C., OL, OM, ON, OV

Ceccotti, Tiberio, OX

Chancé, Antoine, OX

Cherepy, Nerine, OC, OE

Clarke, R. J., OS, OU

Claus, L., OL, OM

Coffee, Ryan, 03

Danly, C., OP

Dayton, Matthew, OL, OM, ON, OV

Dibling, Greg, OF

Dubreuil, Didier, OX

Ducret, Jean-Eric, OX

Durand, C. E., OL, OM

Duval, Alain, $0 X$

Engelhorn, K., OL

England, T., OM

Enomoto, Toshiyuki, $02, \mathrm{OH}, \mathrm{OI}$

Espy, M. A., OA

Fang, L., OM

Fatherley, Valerie E., $\mathrm{OP}, \mathrm{OQ}$

Fittinghoff, David N., $0 Q$

Francis, Manu, 05

Fuchs, Julien, OX

Funsten, Brad, ON

Furenlid, Lars R., 04, 06

Gallegos, Isabel O., OG

Gastineau, Bernard, OX

Gehring, A. E., OA

Geppert-Kleinrath, Verena, OP, OQ

Goodner, Ryan N., OG

Granet, François, OX
Green, J. S., OS

Gregory, C. D., OS

Grim, Gary P., OQ

Guillard, Jean-Christophe, OX

Gupta, Rajiv, OC, OE

Haddock, D., OS

Hagiwara, Osahiko, 02, $0 \mathrm{H}, 0 \mathrm{O}$

Haines, T. J., OA

Harrault, Francis, OX

Heathcote, R. I., OS, OU

Hulin, Sébastien, OX

Hurd, E. R., OL, OM

Jakubowska, Katarzyna, OX

Jimenez, Edward S., OG

Jorgenson, H. Justin, $\mathrm{OQ}$

King, Richard, OF

Kingon, Angus I., OC, OE

Koundinyan, Srivathsan, OG

Kourra, Nadia, OF

Kouts, Katerina, 09

Kusachi, Shinya, 02, OH, Ol

Lantuéjoul, Isabelle, $0 X$

Leboeuf, Didier, OX

Leboeuf, Xavier, OX

Lecherbourg, Ludovic, OX

Levin, Craig S., 03

Loiseau, Denis, OX

Looker, Q., OM

Lotode, Ange, OX

Macaraeg, Chris, ON

Marchet, Bruno, OX

Matsukiyo, Hiroshi, 02, $\mathrm{OH}, \mathrm{Ol}$

McLoughlin, James, OF

Mendoza, E., OP

Miller, S., OD

Mitchell, B. B., OM

Momsen, Neil, 04

Montoya, A., OM

Morioyama, Hodaka, $02, \mathrm{OH}, \mathrm{Ol}$

Morse, Theodore F., OC, $\mathrm{OE}$

Mostovych, Nicholas, OC, OE

Muirhead-Allwood, Sarah, OF

Mukhopadhyay, Sanjoy, 08, 09

Murphy, Timothy, OC, OE

Nagarkar, V. V., OD

Nagel, S. R., OL

Oda, Yasuyuki, 02, OH, Ol

Oertel, John A., $0 Q$

Pès, Chon, OX 
Pickering, Mark R., 05

Porter, J. L., OM

Prunet, Pierre, OX

Rabhi, Nesrine, OX

Raffestin, Didier, OX

Rahman, Tasneem, 05

Reverdin, Charles, OX

Richards, Garrett, 04

Richardson, A. S., OA

Rochau, G., OM

Rossé, Bertrand, OX

Rousseaux, Christophe, OX

Ruiz, T., OV

Ruiz-Gonzalez, Maria, 06

Saluja, Gurdeep, 08

Sanchez, M., OL, OM

Sato, Eiichi, $02, \mathrm{OH}, \mathrm{Ol}$

Sato, Yuichi, $\mathrm{OH}, \mathrm{Ol}$

Schmidt, Derek W., OQ

Segarra, Jose, 08

Selwood, M. P., OU

Sérani, Laurent, OX

Smith, Kilian, 09

Spindloe, C., OS, OU

Stankus, Brian, OC, OE

Suknot, April N., OG

Tafoya, L., OP

Tahtali, Murat, 05

Tao, Li, 03

Thompson, Kyle R., OG

Toussaint, Jean-Christian, OX

Vaughan, J., OP

Vauzour, Benjamin, OX

Vigil, A. J., OM

Volegov, Petr L., OP, OQ

Warnett, Jason M., OF

Wart, M., OD

Watanabe, Manabu, $02, \mathrm{OH}, \mathrm{OI}$

Weber, Peter, OC, OE

Welter, Phillip Vilar, 09

Wilde, Carl, OP, OQ

Williams, Mark A., OF

Yamaguchi, Satoshi, $\mathrm{OH}, \mathrm{Ol}$

Yoshida, Sohei, 02, $\mathrm{OH}, \mathrm{O}$

Zier, J. C., OA 


\section{Conference Committee}

Program Chairs

Ali M. Khounsary, Illinois Institute of Technology (United States)

Ralph B. James, Savannah River National Laboratory (United States)

Conference Chairs

Gary P. Grim, Lawrence Livermore National Laboratory (United States)

Lars R. Furenlid, The University of Arizona (United States)

H. Bradford Barber, The University of Arizona (United States)

Conference Co-chair

Jeffrey A. Koch, National Security Technologies, LLC (United States)

Conference Program Committee

Stuart A. Baker, National Security Technologies, LLC (United States)

Yonggang Cui, Brookhaven National Laboratory (United States)

F. Patrick Doty, Sandia National Laboratories (United States)

Patrick Feng, Sandia National Laboratories (United States)

Paul P. Guss, National Security Technologies, LLC (United States)

Geoffrey Harding, Morpho Detection (Germany)

Khalid M. Hattar, Sandia National Laboratories (United States)

Ralph B. James, Savannah River National Laboratory (United States)

Edward S. Jimenez, Sandia National Laboratories (United States)

Will E. Johns, Vanderbilt University (United States)

Michael J. King, Rapiscan Systems Laboratories (United States)

Edward A. McKigney, Los Alamos National Laboratory (United States)

Wondwosen Mengesha, Sandia National Laboratories (United States)

Frank E. Merrill, Los Alamos National Laboratory (United States)

Rex A. Moats, The University of Southern California (United States)

Vivek V. Nagarkar, Radiation Monitoring Devices, Inc. (United States)

Eiichi Sato, Iwate Medical University (Japan)

Michael R. Squillante, Radiation Monitoring Devices, Inc.

(United States)

Session Chairs

1 Medical Applications: PET/SPECT

H. Bradford Barber, The University of Arizona (United States) 
2 Security and Defense Applications

Lars R. Furenlid, The University of Arizona (United States)

3 Medical Applications: Radiography/CT I

Edward S. Jimenez, Sandia National Laboratories (United States)

4 Medical Applications: Radiography/CT II

Neil Momsen, College of Optical Sciences, The University of Arizona (United States)

5 ICF Diagnostics: Fast X-Ray Imaging

Gary P. Grim, Lawrence Livermore National Laboratory (United States)

6 ICF Diagnostics: Neutron, Gamma, and Other

Jeffrey A. Koch, National Security Technologies, LLC (United States)

7 ICF Diagnostics: Instrumentation

Valerie E. Fatherley, Los Alamos National Laboratory (United States) 